\title{
Understanding the Role of Mirror Neurons in Learning Language: A Review of Studies
}

\author{
Dr. Rishi Mishra ${ }^{1}$, Ashwani Mohan ${ }^{2}$
}

\section{ABSTRACT}

This review aims to understand the implications Rizzolatti's study of mirror neurons in animals to estimate its role in human learning in particular context to language acquisition. Since the mirror neurons are shown to be activated when the subject observes and imitates others in the surrounding, the article further tries to probe the complexity of its functioning when it applies to human beings when compared with animals.

Studies on anosognosia cases have shown that damage to mirror neurons impairs our ability to judge not only other's actions but also their intentions, which points out the significance of mirror neurons in non verbal cue acquisition and hence the role in learning of verbal language. Further studies on EEG in humans have further elaborated the role of mirror neurons in learning in general, after comparing the MU wave activity in the EEG.

Further studies and observations are required understand the functioning of mirror neurons and overcome the limitations surfaced by the earlier studies and also to find out the variations faced in the case of Autistics and individuals with specific learning disability.

Keywords: Mirror Neurons, EEG, Anosognosia.

A mirror neuron is a neuron that fires both when an animal acts and when the animal observes the same action performed by another .Thereby we can say that it "mirrors" the behavior of the other, as though the subject were itself doing the task. Such neurons have been directly observed in primate species. In humans, brain activity consistent with that of mirror neurons has been found in the specific areas namely premotor cortex, the supplementary motor area, the primary somatosensory cortex and the inferior parietal cortex. The function of the mirror system in humans is much more complex and intriguing. Some researchers in cognitive neuroscience and cognitive psychology consider that this mirror neuron provides the physiological mechanism for the perception. It speculates that mirror neurons may be important for understanding the

\footnotetext{
${ }_{1}^{1}$ Amity Institute of Psychology and Allied Sciences, Amity University, Noida, U.P, India

${ }^{2}$ Assistant Professor, AIPS,Amity University, Noida, India

*Responding Author

(C) 2016 I R Mishra, A Mohan; licensee IJIP. This is an Open Access Research distributed under the terms of the Creative Commons Attribution License (http://creativecommons.org/licenses/by/2.0), which permits unrestricted use, distribution, and reproduction in any Medium, provided the original work is properly cited.
} 
actions of other people, and for acquiring new skills by imitation. Mirror neuron systems in the human brain help us acknowledge the actions, feelings and intentions of other people.

\section{METHODOLOGY}

This review is divided into two sections. In the first section, the mechanism and theories of language acquisition are considered ,followed by a review of studies on motor neurons in general is done and their role in language learning is identified. For the purpose of this review, a comprehensive search of the literature was performed

\section{Language Acquisition}

Nativist thinkers (such as Noam Chomsky and Stephen Pinker) believe that certain skills and abilities - such as language learning - are 'hardwired' into the brain.

Modern theories emphasize upon Rational Frame Theory. This theory was developed by Steven C. Hayes and Dermot Barnes-Holmes. This is developed on the concept of B.F. Skinner's Behaviourist ideas, and insists that our language acquisition is purely a result of our interaction with the environment. On the other hand, the Competition model of language acquisition, postulated by Elizabeth Bates and Brian MacWhinney, postulates that the innate cognitive functions are activated by interacting with the environment, allowing us to build up our language abilities.

To conclude we can say that there must be some innate language-learning skills which emphasizes that although children learn between 10 and 15 new words every day, only one of these can be accounted for by direct experience. Noam Chomsky gives a great deal more evidence, saying that almost every sentence that we speak is a brand new combination of words that has never before appeared. To be able to create something like that, the brain must have some kind of programme enabling it to create infinite combinations out of finite resources (ie lists of words).

\section{Mirror neurons may explain speech imitation}

The presence of mirror neurons is consistent with two correspondingly similar theories of speech perception, namely the Motor Theory of speech perception (Liberman, 1957) and the 'articulatory filter hypothesis'( Vihman 2002).

The motor theory of speech perception, postulates that the mental representation of perceived speech is in terms of motor articulatory categories, in dysjunction to acoustic categories. If perception of grasping involves some neurons which are also involved in the performance of grasping, it lends some plausibility to the idea that perception of a spoken particular sound involves neurons which are also involved in the performance of speaking that sound[8] .In these terms, the 'sound' end of the sign relation can be conceived as the junction of a motor schema and a sensory (auditory) schema. Motor schemata are configurations of neurons that, when 


\section{Understanding the Role of Mirror Neurons in Learning Language: A Review of Studies}

activated, produce recognizable precise bodily movements. And a sensory schema is a configuration which, when activated, produces an image of something in the mind. And activation can be half-hearted, as when we just think about hearing a word, or imagine pronouncing it. So the motor theory of speech perception implies, on this 'intersection' view, mirror neurons in the phonetic/phonological representations of words.

A theory such as the motor theory of speech perception could, if true, can be an answer to a problem in language learning. Children are able to reproduce the speech sounds they hear; that is, they somehow know how to sort out their own vocal tracts so as to produce an auditory impression similar to what they hear, even though the unprocessed information reaching the ear is purely acoustic, and not articulatory. Given the motor theory of speech perception, one can understand how a prelinguistic child can so without difficulty learn to reproduce the sound of a word; the acoustic signal is transformed automatically by the ear and brain to a design at least partly expressed in terms of the articulatory movements required for re-pronouncing the word. This link between mirror neurons and the motor theory of speech perception has been emphasized in the literature discussing the potential of the new discoveries to illuminate language evolution (Gallese et al., 1996; Rizzolatti and Arbib, 1998; Skoyles, 1998). The motor theory has certainly not gained wide acceptance, although neither has it been completely ignored. Perception always has to start with a discriminatory incident that is not motoric in nature, but it is believable that automatic motor responses to certain percepts could have evolved.

A theory resembling the Motor Theory of speech perception, though different from it in detail, and possibly overcoming its problems, is the 'articulatory filter hypothesis', proposed by Vihman (1993).

"On this account,the experience of frequently producing CV [consonant-vowel] syllables sensitizes infants to similar patterns in the input speech stream." (Vihman, 2002) What is common to both concepts is the idea that there is some articulatory (i.e. motor) component to children's representations of speech sounds. Vihman (2002) notes the support given to her hypothesis by the discovery of mirror neurons.

Liberman's Motor Theory hypothesizes a strong inherent constituent to the perceptuomotor representation of speech sounds, whereas according to Vihman's articulatory filter hypothesis, the child acquires such representation through familiarity of its own babbling behaviour. Westermann and Miranda (2002) provide an graceful computer replica of the process whereby "mirror neurons responding to both auditory and visual stimuli can develop" (275), based on such feedback from babbling. It is not known if the mirror neurons in the original experimental monkeys develop in ontogeny influenced by the monkey's experience of its own grasping gestures, or whether they are epigenetically programmed to develop in any case, regardless of experience. The innate/acquired issue will concern us again in the conclusion of this paper. 


\section{Understanding the Role of Mirror Neurons in Learning Language: A Review of Studies}

\section{Mirror neurons may aid concept representation}

Likewise the 'sense', concept, or meaning end of the sign relation is neurally the pattern of activation which forms the 'bringing to mind' of a particular concept. Here too, in the theoretical sphere, there are most likely aspects of mirror neuron organization. The central mirror neuron results can most noticeably be applied to the mental representations of bodily actions. For instance, if humans are organized in this respect like macaques, the mental representation of the concept GRASP/GRASPING involves some neurons which are concerned both in the act of grasping and in the observation of grasping.

So thinking of grasping (either by oneself or by someone else) activates these mirror neurons. correspondingly, it seems likely that a representation of the concept WALK/WALKING will involve mirror neurons involved both in the study and the performance of walking. (See the discussion of spontaneous imitative responses in humans in the next section.)

Mirror neurons are, by definition, only involved in the representations of events, such as grasping and walking. Therefore, adhering to a constricted definition of mirror neuron, we cannot claim that the mental representations of objects, such as apples and screwdrivers, involve mirror neurons. Apples and screwdrivers are not actions. But it seems likely that representations of objects involve some analogy between motor and sensory neurons, similar to that found in the representations of actions. Attending to or acting on a real apple in an appropriate way, or imagining an apple, involves bringing to mind the notion of an apple. The mental representations of tools involve areas of motor cortex apt for handling them, beside sensory information about what the apparatus look like (Martin et al., 1996). It is hard to disconnect the passive physical feel of an object from active knowledge of what to do with it. Similarly, one's concept of, say, an apple, includes motor information about how to hold it and bite it, as well as sensory information about what it looks/tastes/smells like (Fadiga et al., 2000; Murata et al., 1997).

Mentally simulating the actions of others is thought to be a key component of empathy. Yet new research suggests that our so-called mirror neurons may also expose hidden divisions. A study published in October in the journal PLOS ONE reveals that these copycat neurons do not reflect all people equally.

Mirror neurons were discovered in the early 1990s, and their existence was a neuroscientific revelation: brain cells not only fire when we perform a given action, they also fire when we see someone else doing the deed. Much later work has suggested that mirror neurons undergird social cognition. Now budding research is finding that our mirror neuron system distinguishes between people who are physically and culturally similar and those who are not. The new work probed these differences further. In the study, investigators asked 17 young adult Jewish men to review the biographies and photographs of eight individuals who physically resembled the participants. Half these characters, portrayed by actors, were identified as neo-Nazis. The subjects reported they strongly disliked the anti-Semitic characters but not the others. Next, the 
participants underwent efficient MRI scans while watching a video of each character drinking from a water bottle. The researchers focused on the ventral premotor cortex, a region typically active when we carry out an action or watch someone else do so. They found that neurons in this region activated differently when subjects viewed detestable and likable characters.

Since mirror neuron activity is thought to be a very basic part of brain function-and it can be seen in many animals besides humans - the new finding supports the notion that our brain is predisposed to distinguish "us versus them." This distinction can be beneficial, encouraging caution around those with harmful intentions, or dangerous, further entrenching prejudices. To weaken unwelcome biases, lead author Lisa Aziz-Zadeh, a cognitive neuroscientist at the University of Southern California, suggests that exposure and perspective taking could go a long way.

\section{THE EMERGENCE OF LANGUAGE}

Rizzolatti's discovery can help us solve this complex situation. He recorded from the ventral premotor area of the frontal lobes of monkeys and found that certain cells will fire when a monkey performs a single, highly specific action with its hand: pulling, pushing, tugging, grasping, picking up and putting a peanut in the mouth etc. different neurons fire in response to different actions.[9] One might be tempted to think that these are motor "command" neurons, making muscles do certain things; though, the surprising truth is that any given mirror neuron will also fire when the monkey in question observes another monkey (or even the experimenter) performing the same action, e.g. tasting a peanut! By knowing about these neurons, you have the basis for understanding a host of very enigmatic aspects of the human mind: "mind reading" empathy, imitation learning, and even the evolution of language.

When you watch someone else doing something (or even starting to do something), the corresponding mirror neuron might fire in your brain, thereby allowing you to "read" and understand another's intentions, and thus to develop a sophisticated "theory of other minds." (I suggest, also, that a loss of these mirror neurons may explain autism. Without these neurons the child can no longer understand or empathize with other people emotionally and therefore completely withdraws from the world socially.)

Mirror neurons can also facilitate you to reproduce the movements of others thereby setting the stage for the complex Lamarckian that characterizes our species and liberates us from the constraints of a purely gene based evolution. Moreover, as Rizzolati has noted, these neurons may also enable you to mime - and possibly understand - the lip and tongue movements of others which, in turn, could provide the opportunity for language to evolve. Once we have these two abilities in place the ability to read someone's intentions and the ability to mime their vocalizations then it will set in motion the evolution of language. 
Another important piece of the puzzle is Rizzolatti's observation that the ventral premotor area may be a homologue of the "Broca's area" - a brain center associated with the expressive and syntactic aspects of language in humans.

These arguments do not in any way negate the idea that there are specialized brain areas for language in humans. We are dealing, here, with the question of how such areas may have evolved, not whether they exist or not.

Mirror neurons were discovered in monkeys but how do we know they exist in the human brain? To find out we studied patients with a strange disorder called anosognosia. Most patients with a right hemisphere stroke have complete paralysis of the left side of their body and will complain about it, as expected. But about $5 \%$ of them will vehemently deny their paralysis even though they are mentally otherwise lucid and intelligent. This is the so called "denial" syndrome or anosognosia.

To our amazement, we found that some of these patients not only denied their own paralysis, but also denied the paralysis of another patient whose inability to move his arm was clearly visible to them and to others. Denying ones one paralysis is odd enough but why would a patient deny another patient's paralysis? We suggest that this bizarre observation is best understood in terms of damage to Rizzolatti's mirror neurons. It's as if anytime you want to make a judgement about someone else's movements you have to run a VR (virtual reality) simulation of the corresponding movements in your own brain and without mirror neurons you cannot do this .

The second piece of support comes from studying brain waves (EEG) in humans. When people move their hands a brain wave called the MU wave gets blocked and disappears completely. Eric Altschuller, Jamie Pineda, and I suggested at the Society for Neurosciences in 1998 that this suppression was caused by Rizzolati's mirror neuron system. Consistent with this theory we found that such a suppression also occurs when a person watches someone else moving his hand but not if he watches a similar movement by an inanimate object. (We predict that children with autism should show suppression if they move their own hands but not if they watch some one else. (Social Neuroscience Abstracts 2000).

\section{LIMITATIONS}

Most of the research on mirror neurons has been conducted in monkeys.( Wired Christian Jarrett, 2013 ) To date, there are no widely accepted neural or computational models to describe how mirror neuron activity supports cognitive functions[10].

\section{REFERENCE}

Dinstein I, Thomas C, Behrmann M, Heeger DJ (2008). "A mirror up to nature". Curr Biol 18 (1): R13-8. doi:10.1016/j.cub.2007.11.004.PMC 2517574. PMID 18177704. 
Dinstein I, Thomas C, Behrmann M, Heeger DJ ,2008)

Heyes, Cecilia (2009). "Where do mirror neurons come from?".Neuroscience and Biobehavioral Reviews.

Hickok, G. (July 21, 2009). "Eight Problems for the Mirror Neuron Theory of Action Understanding in Monkeys and Humans". Journal of Cognitive Neuroscience 7:12291243.

Jarrett,Christian,A Calm Look At The Most Hyped Concept In Neuroscience-Mirror Neurons, Wired,12.13.13,[1]

Molenberghs P, Cunnington R, Mattingley J (July 2009). "Is the mirror neuron system involved in imitation? A short review and meta-analysis.". Neuroscience Biobehavioral Reviews 33 (1): 975-980

Rizzolatti, G. and Arbib, M.A. (1998) Language within our grasp. Trends in Neuroscience, 21:188-194

Rizzolatti, Giacomo; Craighero, Laila (2004). "The mirror-neuron system". Annual Review of Neuroscience 27 (1): 169-192.

Rizzolatti, Giacomo; Fadiga, Luciano (1999). "Resonance Behaviors and Mirror Neurons". Italiennes de Biologie 137: 85-100

Saussure, F.d. (1959) Course in General Linguistics. New York: The Philosophical Library. Translated by Wade Baskin

How to cite this article: R Mishra, A Mohan (2016), Understanding the Role of Mirror Neurons in Learning Language: A Review of Studies, International Journal of Indian Psychology, Volume 3, Issue 3, No. 9, DIP: 18.01.162/20160303, ISBN: 978-1-365-13820-1 